\title{
Variations in Position of Conus Medullaris in Adult Patients
}

\author{
Ayoob Rostamzadeh,,$^{1,}$ Farhad Naleini, ${ }^{2}$ and Omid Rostamzadeh ${ }^{3}$ \\ ${ }^{1}$ Department of Anatomical Sciences, Shahrekord University of Medical Sciences, Shahrekord, IR Iran \\ ${ }^{2}$ Department of Radiology, Kermanshah University of Medical Sciences, Kermanshah, IR Iran \\ ${ }^{3}$ Department of Occupational Therapy, Faculty of Rehabilitation, Iran University of Medical Sciences, Tehran, IR Iran \\ "Corresponding author: Ayoob Rostamzadeh, Department of Anatomical Sciences, Shahrekord University of Medical Sciences, Shahrekord, IR Iran. E-mail: \\ ayoobrostamzade@gmail.com
}

Received 2015 February 3; Accepted 2015 November 17.

Keywords: Conus Medullaris, Surgical Procedures, Patients

\section{Dear Editor,}

Finding a safe location for spinal block during surgical procedures is a matter that requires attention. Conus medullaris (CM) is the most distal point of the spinal cord and dura that could be visualized on the sagittal sequence. $\mathrm{CM}$ location follows a normal distribution, from the lower third of L1 (ranging from the middle third of T12 to the upper third of L3) [1]. It is important to know the level of the lumbar spine to have a safe spinal block after an intervertebral injection. Counting the intervertebral spaces before spinal block is an important way to evade damage to the spinal cord. The neurological structures at the level of the thoracolumbar spine are critical for lower-extremity motor, sensory function, as well as bowel, bladder and sexual functions [2]. Purpose of this study was to find relationship between the location of CM and spinal position in adult patients, with age, gender and body mass index (BMI). This study was conducted on patients referring to Shahid Chamran MRI center in Sanandaj, West of Iran. For this study 350 patients (199 males and 151 females) were selected. T1-weighted sagittal spin echo MRI sequences of the lumbar spine with the patient in the supine position were studied to evaluate the location of CM. SPSS-20 was applied for the statistical analysis using $\chi^{2}$ and ANOVA tests. The $\mathrm{P}$ values are at a significant level of $\mathrm{P}<0.05$. The position of the CM was between T12 upper third and L2 middle third with a mean of L1 upper third. The conus was located from T12 upper third to L2 upper third (mean: L1 upper third) in males and from T12 upper third to L2 middle third (mean: L1 middle third) in females. In different age groups, the mean position was variable ranging from T12 - L1 intervertebral disc to upper part of L1 middle third and did not seem to be clinically significant. Saifuddin et al. [3] in a study, with 504 adult cases without spinal deformity, found that the tip of the conus was between middle third of T12 and upper third of L3 with a mean position at the lower third of L1. They found that conus position was slightly lower in males than in females without statistical significance. The results of this study are congruent with our study. In contrast to the findings of Saifuddin et al. the results of our study did not show a significant difference between CM position in males and female [3]. The present study showed that it would be practical to know the CM position in the same patient population; since lumbar radiograph and MRI cannot be routinely recommended for all patients scheduled for spinal block. It is important to consider the possible range of CM level when performing lumbar puncture and avoid complications for lumbar surgery. Finally, the variation of the CM position could be a guidance that why neurological symptoms varies for different patients.

\section{References}

1. Demiryurek D, Aydingoz U, Aksit MD, Yener N, Geyik PO. MR imaging determination of the normal level of conus medullaris. Clin Imaging. 2002;26(6):375-7. [PubMed: 12427430].

2. Harkey H3, White E, Tibbs RJ, Haines DE. A clinician's view of spinal cord injury. Anat Rec B New Anat. 2003;271(1):41-8. doi: 10.1002/ar.b.10012. [PubMed: 12619085].

3. Saifuddin A, Burnett SJ, White J. The variation of position of the conus medullaris in an adult population. A magnetic resonance imaging study. Spine (Phila Pa 1976). 1998;23(13):1452-6. [PubMed: 9670396]. 